\title{
Circulating miRNAs profiles in tourette syndrome: molecular data and clinical implications
}

Renata Rizzo ${ }^{1 \dagger}$, Marco Ragusa ${ }^{2 \dagger}$, Cristina Barbagallo², Mariangela Sammito², Mariangela Gulisano ${ }^{1}$, Paola V Cali ${ }^{1}$, Claudio Pappalardo², Martina Barchitta ${ }^{3}$, Mariagrazia Granata ${ }^{2}$, Angelo G Condorelli², Davide Barbagallo², Marina Scalia ${ }^{2}$, Antonella Agodi ${ }^{3}$, Cinzia Di Pietro ${ }^{2}$ and Michele Purrello $0^{1,4^{*}}$

\begin{abstract}
Background: Tourette Syndrome (TS) is a highly prevalent childhood neuropsychiatric disorder (about $1 \%$ ), characterized by multiple motor and one or more vocal tics. The syndrome is commonly associated to comorbid conditions (e.g., Attention Deficit Hyperactivity Disorder and Obsessive Compulsive Disorder), which considerably aggravate clinical symptoms and complicate diagnosis and treatment. To date, TS molecular bases are unknown and its molecular diagnosis is unfeasible.
\end{abstract}

Results: Due to their master role within cell networks and pathways both in physiology as in pathology, we sought to determine the transcriptome of circulating miRNAs in TS patients: by TaqMan Low Density Arrays, we profiled the expression in serum of 754 miRNAs in six TS patients and three unaffected controls (NCs) (discovery set). These data were validated by single TaqMan assays on serum from 52 TS patients and 15 NCs (validation set). Network and Gene-ontology analysis were performed by using Cytoscape and Babelomics server. We found that miR-429 is significantly underexpressed in TS patients with respect to NCs. Decreased serum levels of miR-429 allowed us to discriminate TS patients from NCs with $95 \%$ of sensitivity and $42 \%$ of specificity. Intriguingly, computational analysis of the network comprising miR-429 targets demonstrates their involvement in differentiation of midbrain and hindbrain and synaptic transmission.

Conclusions: Our data open the way to further molecular characterization of TS and eventual identification of the corresponding genotypes. Circulating miR-429 may be immediately useful as sensitive molecular biomarker to support TS diagnosis, actually based only on DSM-V criteria.

Keywords: Tourette syndrome, Circulating miRNAs, Molecular pathogenesis, Molecular biomarkers, Minimally invasive molecular diagnosis

\section{Background}

Gilles de la Tourette Syndrome (TS) is a highly prevalent childhood neurodevelopmental disorder, characterized by both multiple motor and vocal tics: these appear during illness, not necessarily concurrently [1]. Tics are sudden, rapid, recurrent, non rhythmic movements or vocalizations, which wax and wane in frequency; to be

\footnotetext{
*Correspondence: purrello@unict.it

${ }^{\dagger}$ Equal contributors

'Section of Child Neurology and Psychiatry, Department of Experimental and Clinical Medicine, University of Catania, Catania, EU, Italy

${ }^{4}$ Via S Sofia 87, Building C, $2^{\circ}$ floor, room 10, 95123 Catania, EU, Italy

Full list of author information is available at the end of the article
}

diagnostically useful, they must have persisted for more than 1 year since onset (Diagnostic and Statistical Manual of Mental Disorders, DSM-V) [1, 2]. TS prevalence is estimated to be about $1 \%$ [2]. The syndrome is commonly associated to comorbid conditions as Attention Deficit Hyperactivity Disorder (ADHD) and Obsessive Compulsive Disorder (OCD) [2], which have been reported in 60, and $45-60 \%$ of TS patients, respectively [3]: both ADHD and $\mathrm{OCD}$ sensibly impair their health-related quality of life [4-6]. The most disabling of them is OCD, which also causes poor self-esteem and low quality of life [7]. It has been stressed that these comorbidities should be 
considered as negative prognostic factors, given that they considerably worsen prognosis [7-9]. Different TS symptoms are associated with dysfunctions of distinct cortical areas: this has clear implications for the neuroanatomical model of the syndrome [10]. TS has a multifactorial etiology, in which genetic, neurological and environmental factors interact to determine the clinical phenotype [1]. Several genetic mutations have been suggested to be involved in TS (e.g., those at the SLITRK1 locus or at loci of genes belonging to dopaminergic and serotoninergic pathways) [1, 11]. MicroRNAs (miRNAs) are small non protein-coding RNAs (19-25 nucleotides long), which negatively regulate gene expression at the posttranscriptional level $[12,13]$. MiRNAs are master regulators of cellular pathways and networks: accordingly, they perform key roles in different biological processes and diseases $[14,15]$. Serum/circulating miRNAs (cmiRNAs) have been detected in all body fluids analyzed to date $[16,17]$ : this discovery has disclosed the possibility of identifying minimally invasive biomarkers of disease through liquid biopsies [18]. In psychiatric diseases, this approach has been already applied to Autism [19], Bipolar Disorder [20], Schizophrenia [21], Depression [22], but not to TS. We hypothesized that altered cmiRNAs serum profiles could be identified also in TS and hint to its biomolecular bases; furthermore and equally important, these molecular data could be immediately applied to strengthen traditional TS diagnosis, actually based on DSM-V criteria. Through a high throughput approach, we identified a cmiRNA differentially expressed (DE) in sera from TS patients with respect to healthy controls. After performing a computational analysis of its target mRNAs, we discovered that these encode proteins that are involved in differentiation of midbrain and hindbrain and synaptic transmission.

\section{Results}

\section{Demographic data}

Fifty eight young caucasian people affected by TS [mean age 12.7 y $(\mathrm{SD} \pm 0.9)$, sex $\mathrm{M}: \mathrm{F}=50: 8]$ and 18 unaffected controls (NCs) [mean age 12.2 y $(\mathrm{SD} \pm 0.9)$, sex $\mathrm{M}: \mathrm{F}=$ 14:4] were recruited into the study. Age distribution was not significantly different within the TS cohort respect to NCs ( $p$-value: 0.170$) .87 .5 \%$ of TS patients and

Table 1 Clinical features

\begin{tabular}{llll}
\hline & TS & NCs & $p$-value* \\
\hline Sex M:F & $50: 8$ & $14: 4$ & 0.178 \\
Mean age & $12.7( \pm 0.9)$ & $12.2( \pm 2.1)$ & 0.170 \\
Mean age at onset & $5.9( \pm 1.91)$ & & \\
Ethnic Background: Caucasian & 58 & 18 &
\end{tabular}

TS: Tourette Syndrome

NC: Normal Controls

Standard Deviation is shown between parenthesis

*statistical significance $p<0.05$
73.3 \% NCs were males: also this difference is not statistically significant ( $p$-value 0.178 ) (Table 1 ).

\section{Neuropsychiatric findings in TS patients}

All TS patients presented DCI > $80 \%$ (mean score $87.6 \%$, $\mathrm{SD} \pm 7.6$ ). Concerning YGTSS (Yale Global Tic Severity Rating Scale), patients presented a mean total tic score of $29.7(\mathrm{SD} \pm 7.1)$ and a mean impairment score of 27.9 (SD \pm 8.7 ). The mean C-YBOCS (Children's Yale-Brown Obsessive Compulsive Scale) score was $18.3(\mathrm{SD} \pm 8.7$ ) (Table 2). Many patients (39 out 58:67 \%) required pharmacological treatment with SSRI, neuroleptics or both. We detected a statistically significant difference in CADS (Conner's ADHD/DSMV-IV Scale) score between TS patients and NCs ( $p$-value 0.000) (Table 2).

\section{MiRNAs profile in TS patients}

Through TaqMan Low Density Array (TLDA) analysis, we determined the total expression profiles of 754 miRNAs in sera from six TS patients and three unaffected controls. Real time experiments were separately performed for fluidic card A and B: each card contained 384 TaqMan assays. Cycle thresholds (Cts) obtained for each sample for each miRNA were used to calculated DCt, which were computed by the SAM method to identify differentially expressed (DE) miRNAs (Additional file 1). We found that only miR-429 was differentially expressed: it was significantly downregulated in serum of TS patients with respect to NCs by using both GMN method and miR-320 as endogenous control $(\mathrm{FDR}=0.024)$.

\section{Validation by single TaqMan assays}

Expression of miR-429 was subjected to validation through single TaqMan assays in serum of 52 TS patients and 15 NCs. By applying both parametric ( $t$-test) and non parametric (Wilcoxon test) tests, we confirmed the statistically significant downregulation of miR-429 in TS patients with respect to $\mathrm{NCs}$ (Wilcoxon test $p$-value $=0.01$; $t$-test $p$-value $=0.004)($ Fig. 1). By computing a ROC curve, we found that the decrease of miR-429 serum levels was able to discriminate TS patients from NCs. Specifically, we obtained an AUC of 0.75 (95\% CI, 0.584-0.907; $p=0.01$ ), with $95 \%$ of sensitivity and $42 \%$ of specificity (DCt cutoff value: 10.32) (Fig. 2).

\section{Correlation between miR-429 expression and neuropsychiatric parameters}

To evaluate whether expression changes of miR-429 were associated with the clinical and pathologic characteristics observed in patients, its expression levels were compared to different parameters including C-YBOCS, YGTSS, Conner's ADHD. However, we found only a weak positive correlation between Conner's ADHD and miR-429 expression (Pearson $=0.28$; $p$ value $=0.03$ ), while 
Table 2 Neuropsychological findings

\begin{tabular}{llll}
\hline & TS & NC & -value \\
\hline DCl & $88.7 \%( \pm 7.8)$ & & \\
YGTSS & & & \\
$\quad$ Total Tic Score & $29.7( \pm 7.1)$ & & \\
$\quad$ Impairment & $27.9( \pm 8.7)$ & & \\
C-YBOCS & $18.3( \pm 8.7)$ & & 0.000 \\
CONNERS & $18.8( \pm 2.7)$ & $7.5( \pm 2.4)$ & 0.370 \\
CDI & $8.6( \pm 5.4)$ & $7.3( \pm 2.6)$ & 0.478 \\
MASC & $44.8( \pm 13.7)$ & $41.8( \pm 17.2)$ & \\
CBCL & & & 0.000 \\
Tot & $32.7( \pm 7.4)$ & $22.1( \pm 6.2)$ & 0.028 \\
Int & $9.9( \pm 2.9)$ & $8.1( \pm 2.2)$ & 0.002 \\
Ext & $10.9( \pm 2.6)$ & $8.8( \pm 2.7)$ & \\
\hline
\end{tabular}

DCl: Diagnostic Confidence Index

YGTSS: Yale Global Tic Severity Scale

C-YBOCS: Children-Yale Brown Obsessive Compulsive Scale

CDI: Child Depression Inventory

MASC: Multidimensional Anxiety Scale for Children

CBCL: Child Behavior Check-List; Tot: Total; Int: Internalizing; Ext: Externalizing

Standard Deviation is shown between parenthesis

*statistical significance $p<0.05$

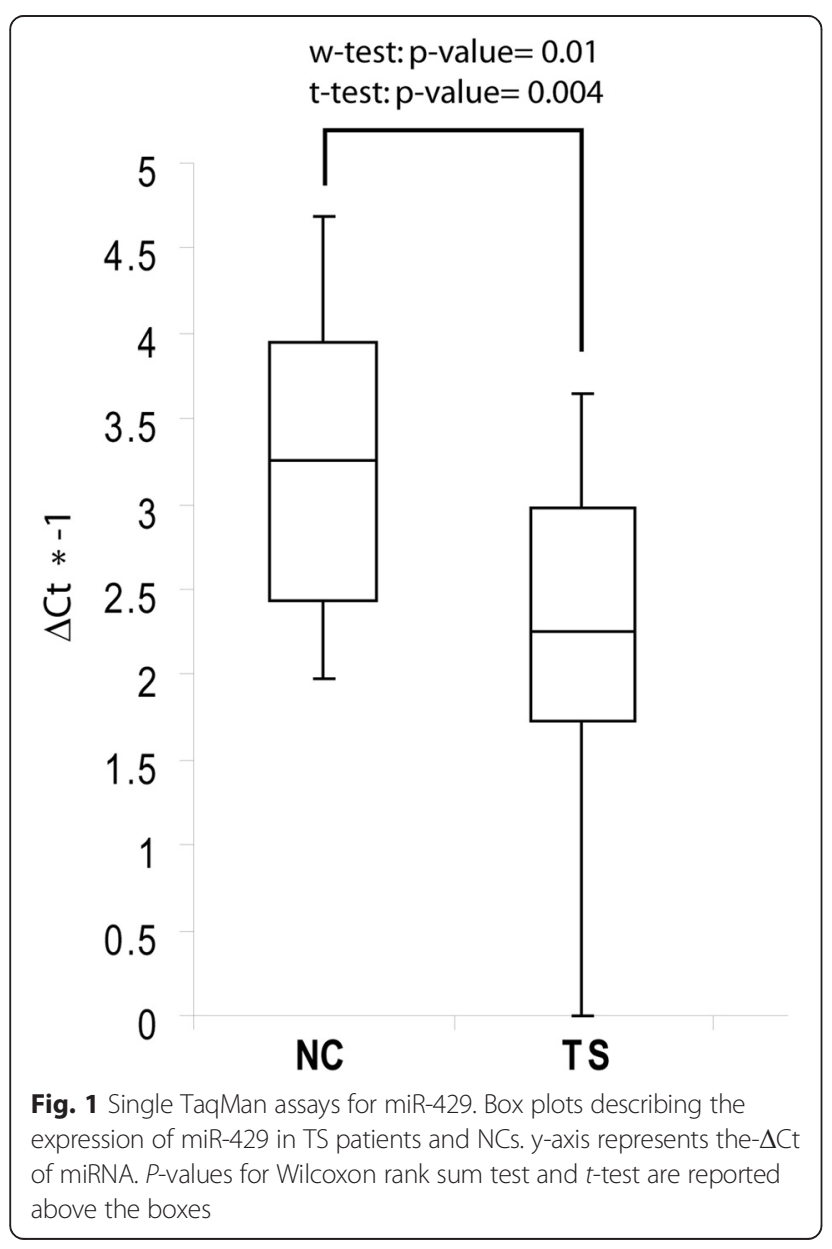

there were no significant associations among miRNAs expression and C-YBOCS and YGTSS scores. Next, we assigned the expression levels of miR-429 to comorbidity groups: (TS + OCD: 20 patients), (TS + ADHD: 13 patients), (TS + ADHD + OCD: 16 patients). We compared the expression of miR-429 among different comorbidity groups and patients with no comorbidity (Fig. 3). We found no statistically significant difference of expression among these groups, although patients with TS+ADHD+OCD showed a downregulation of miR-429 slightly more pronounced than other groups.

\section{MiRNA targets identification and network analysis}

To pinpoint the biological functions of miR-429 and rationalize its etiological involvement in TS, we computationally retrieved its validated targets (26 targets based on TarBase); we then built a molecular network comprising miR-429 validated targets and their first neighbours. The generated network, consisting of 1472 nodes and 11,532 edges, was topologically centred on BCL2, CREBBP, EP300, HNF4A, MYC (network nodes with highest degree and betweenness). By using FatiGO enrichment analysis tool, we compared the statistically overrepresented biological functions of all nodes within the network against the entire genome; the following databases were analyzed: Biocarta, Reactome, KEGG, GO. We found that miR-429 directly or indirectly interacts with proteins involved in processes related to physiological and pathological functions of the nervous system (e.g., development and differentiation of neurons, synaptic and postsynaptic transmission and plasticity, neurodegenerative diseases), (Hypergeometric test; Benjamini \& Hochberg FDR Correction; $p \leq 0.01$ ) (Fig. 4).

\section{Discussion}

Within cells, miRNAs perform a critical biomolecular role as master regulators of networks and pathways. $\mathrm{Nu}-$ merous studies have demonstrated that the genes encoding them are causally implicated in neurodegenerative diseases (e.g., Parkinson's Disease [23], Alzheimer's Disease [24], Huntington's Chorea [25], Frontotemporal Dementia [26]). They also are causally involved in neuropsychiatric disorders (e.g., Schizophrenia [27], Bipolar Disorder [28], Autism [29]). Circulating miRNAs have been already reported as potential markers of these diseases [18-21]. Tourette syndrome was the first neuropsychiatric disease etiologically linked to miRNAs, when TS patients were reported to have a mutation in the binding site of miR-189 at the 3' UTR of the SLITRK1 gene [30]. This finding was not followed by further characterization of miRNAs role in TS; furthermore, no data on cmiRNAs related to TS have been published to date. Notwithstanding the uncertainty on their tissues of origin, the discovery of cmiRNAs has opened new exciting 


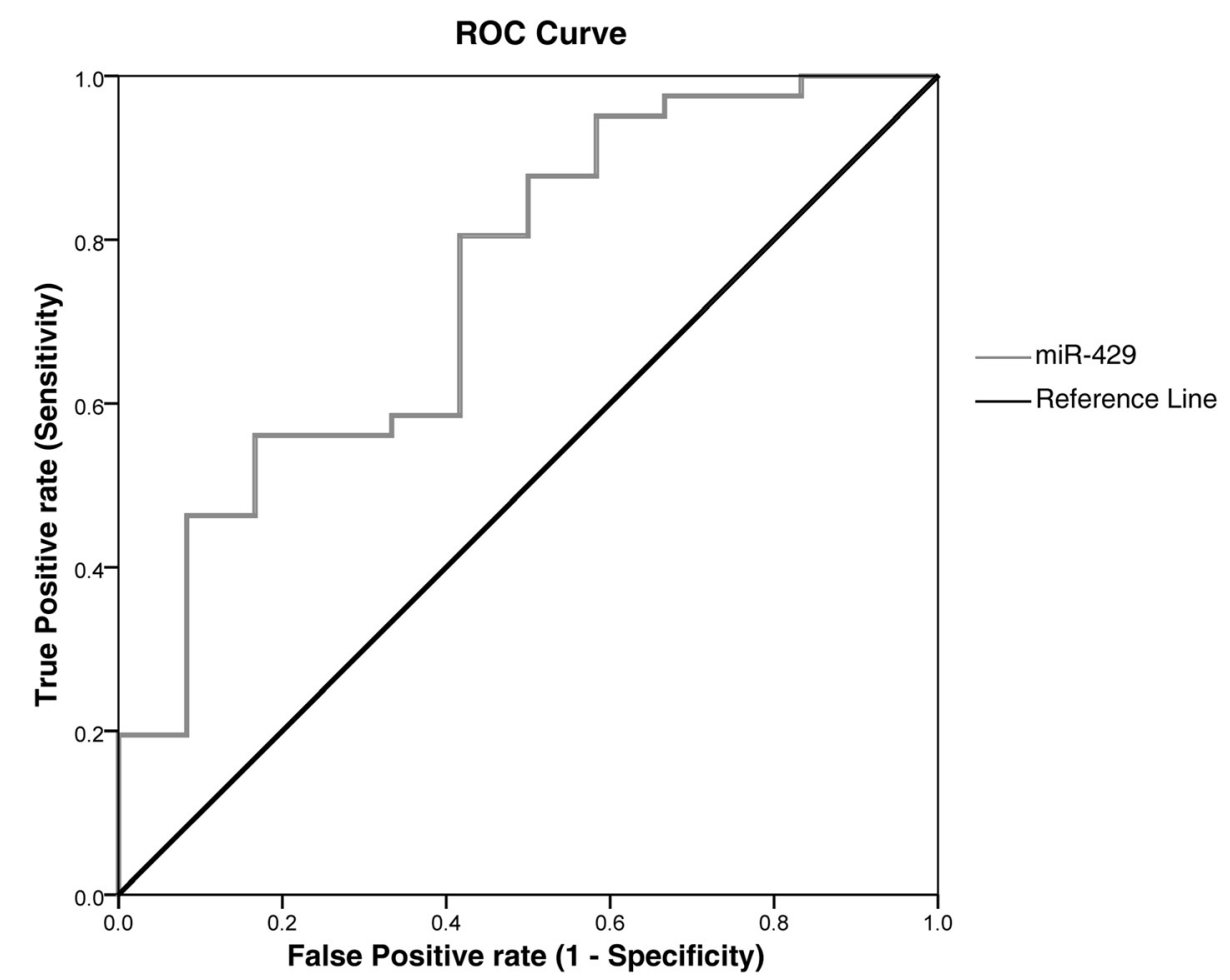

Fig. 2 Receiver Operator Characteristic (ROC) Curve for miR-429 expression in TS patients. ROC curve of miR-429 DCts for TS detection. Gray curve represents DCts calculated by using miR-320 as endogenous control

research perspectives for the identification of the biomolecular bases of pathologic phenotypes [16, 31, 32]; it also promises important translational applications to clinical diagnosis and therapy [33, 34]. In fact, cmiRNAs are endowed with many characteristics that confer them the properties of good biomarkers. They are easily detectable by commonly applied analytical methods as qRTPCR [34]. They also are much more stable than
mRNAs in body fluids, possibly due also to their inclusion in nucleoprotein complexes [35] or encapsulation in microvescicles and exosomes [36, 37]: accordingly, cmiRNAs are resistant to harsh condition, as multiple cycles of freezing - thawing, boiling, very low or high $\mathrm{pH}$, extended storage. Profiling data on cmiRNAs in patients with neuropsychiatric disorders have been already reported for bipolar disorder [20], schizophrenia [21] and depression

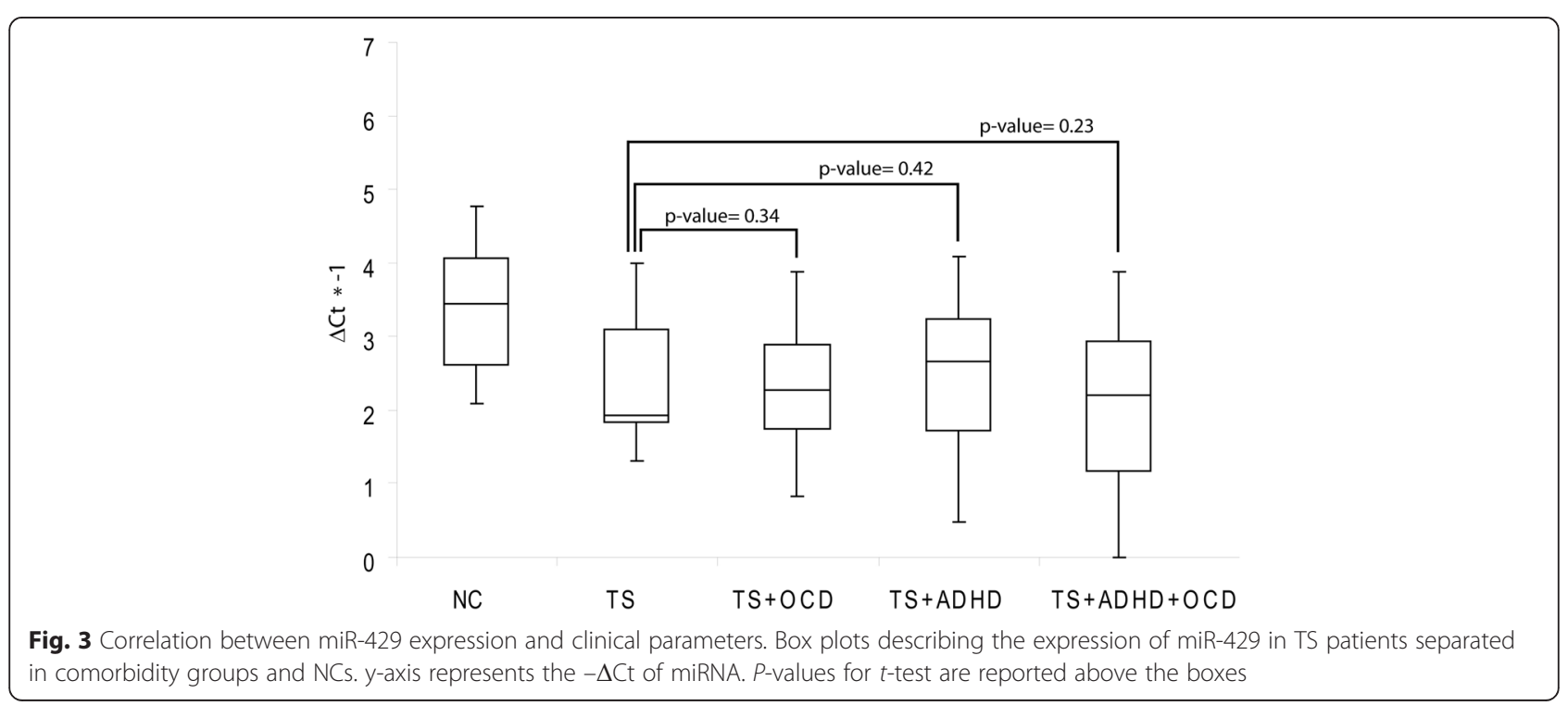




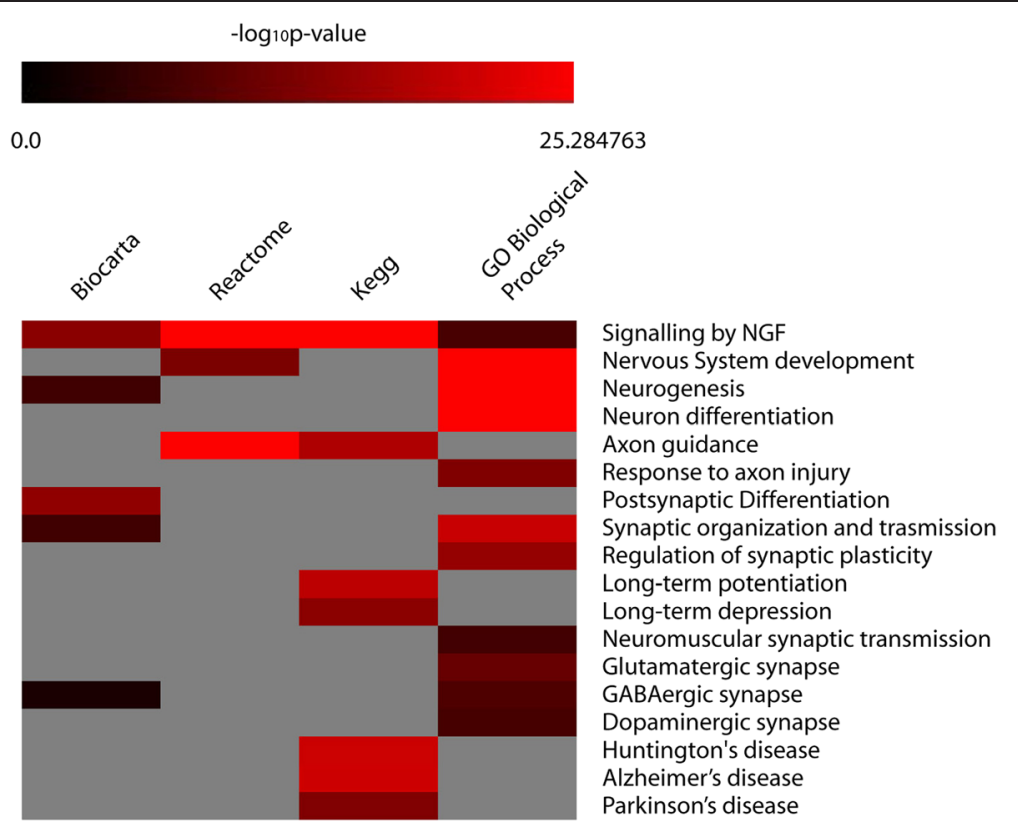

Fig. 4 Biological Processes controlled by miR-429 network. Overrepresented biological functions from a molecular network built on validated miR-429 targets, retrieved by different annotation databases (Biocarta, Reactome, KEGG, GO). Data are shown as - log10 of p-values for each biological process

[22]. For these pathologies, clinical diagnosis is currently based on DSM-V criteria (Diagnostic and Statistical Manual of Mental Disorders, fifth edition): profiling of cmiRNAs, after a liquid biopsy, offers a new potentially very important molecular diagnostic tool. Our study is the first report on profiling of miRNAs transcriptome in serum from TS patients. Our experiments allowed us to identify the statistically significant downregulation of cmiR-429 in serum from TS patients: this allowed us to discriminate TS patients from unaffected controls. In fact, ROC curve analysis suggests that this miRNA could be considered a good marker to diagnose TS, as its sensitivity is $95 \%$ and its specificity is $42 \%$. Finally, our data show a statistically significant positive association between miR-429 expression and Conner's ADHD, suggesting a molecular relationship between biological processes regulating attentional control and inhibitory control (e.g., dopamine and norepinephrine pathways) and circulating miRNA expression. MiR-429 is a member of miR-200 family (i.e., miR-141, -200a, -200b, -200c, miR-429), which has been shown to inhibit the epithelial-mesenchymal transition by downregulating ZEB1 and ZEB2 $[38,39]$. Interestingly, the genes encoding miR-200a, miR-200b and miR-429 itself are located at $1 \mathrm{p} 36.33$, a genomic region with copy number variants that has been recently associated to Autism [40, 41]. The potential involvement of miR-429 in Autism is intriguing, as it has been suggested that TS, ADHD, ASD (Autism Spectrum Disorders), and OCD share a common genetic background [42, 43].
By directly targeting the pluripotency factor Sox 2 and the cell-cycle regulator E2F3 in neural stem/progenitor cells, members of miR-200 family promote cell-cycle exit and neuronal differentiation of ventral midbrain/hindbrain neural progenitors, including midbrain dopaminergic neurons [44]. Further, data on mice treated with cocaine suggest that the expression of miR-429 could be involved in dendritic morphology and synaptic plasticity [45]. Altogether, these data strongly suggest that miR-429 may be involved in molecular circuits, which control several aspects of midbrain and hindbrain differentiation and synaptic transmission: this is nicely highlighted by our computational analysis of the molecular network centered on miR-429 targets (Fig. 4). The potential importance of these results is stressed by the results of several studies, which have reported alterations in TS of molecular pathways controlling regional boundary formation during brain development, including their essential role in midbrainhindbrain boundary formation [46-48], and proposed that dopaminergic and serotonergic genes are involved in TS etiopathogenesis [49].

\section{Conclusions}

Based on our data, we propose that miR-429 is a promising biomarker for minimally invasive molecular diagnosis of TS: accordingly, it should be added to classical diagnostic clinical methodologies. Interestingly, our network analysis shows that miR-429 targets are involved in critical aspects of neurodifferentiation and synaptic 
transmission: these findings open the way to further molecular characterisation of TS and eventual identification of TS genotypes.

\section{Methods}

\section{Ethics Statement}

This study was conducted according to the Declaration of Helsinki and was approved by the Institutional Review Board of the University of Catania; a written informed consent was obtained from each study participant.

\section{Patients recruitment}

Peripheral blood samples were collected from 58 young Caucasian people affected by TS [age range 6-17 y; mean age 12.7 y $(S D \pm 0.9)$, sex $\mathrm{M}: \mathrm{F}=50: 8]$ and compared with $18 \mathrm{NCs}$ [age range 10-15 y; mean age $12.2 \mathrm{y}$ $(\mathrm{SD} \pm 0.9)$, sex $\mathrm{M}: \mathrm{F}=14: 4]$. TS patients presented an age of onset of 5.9 years $(\mathrm{SD} \pm 1.91)$ : they were recruited and clinically studied at the Neuropsychiatric Unit, Section of Child Neurology and Psychiatry, Department of Clinical and Experimental Medicine, University of Catania, Catania, Italy, EU. NCs were volunteers recruited from local schools. Demographics are reported in Table 1.

\section{Neuropsychological procedures}

Upon collecting basic demographic data, diagnosis of TS and associated clinical conditions was made in accordance to DSM-V criteria by child neurologists with extensive specific experience (RR and her Collaborators). All TS patients were initially assessed by using a semistructured interview (i.e., the National Hospital Interview Schedule for Gilles de la Tourette Syndrome) [50] and DCI [51]. Patients with TS and NCs completed the WISC-III [52], YQOL-R [53], MASC [54], CDI [55], CADS [56], and the Child Behavior Checklist (CBCL) [57]. MASC and CDI were used to assess anxiety and depression, CADS provided an indication of symptoms related to $\mathrm{ADHD}$, and $\mathrm{CBCL}$ was used to assess a range of emotional and behavioral difficulties. TS patients underwent two additional clinician-rated interviews: the YGTSS [58], to measure the severity of tics, and the Y-BOCS [59] to assess symptoms related to OCD. The YGTSS is an 11-item clinician-rated interview of motor and phonic tic severity. The clinician initially notes the presence of motor and phonic tics based on child and parent report over the past week, as well as behavioral observations. Following this, the clinician rates the severity of motor and phonic tics on five separate dimensions each: number, frequency, intensity, complexity and interference. These scales score rating from 0 to 50 . The YGTSS also includes a separate impairment rating scale also rated from 0 to 50. Higher scores indicate the presence of severe symptoms and impairment. The CY-BOCS is the most used and reproducible instrument to assess the severity of obsessive-compulsive symptoms in children. The clinician initially notes the presence of obsessions and compulsions based on child and parent report over the past week, as well as behavioral observations. Following this, the clinician rates the severity of obsessions and compulsions on separate dimensions each: number, frequency, intensity, resistance and interference. Three index scores are obtained: Obsession score, Compulsion score and Total score. The total score evaluates the impairment that obsessive - compulsive symptoms caused to the patient. Based on the total score, the results can be interpreted as follows: 0-7 sub-clinical; 8-15 mild; 16-23 moderate; 24-31 severe; 32-40 extreme. Patients scoring in the mild range or higher are likely experiencing a significant negative impact on their quality of life.

\section{Entry criteria}

We included in this study TS patients who presented normal IQ (tested with Wechsler Scale). Initial diagnosis was confirmed after one year: patients who were unable to be fully assessed at the initial evaluation and/or at follow-up were excluded. Moreover, we excluded all patients who showed evidence of severe neurological or physical impairment. Our cohort was comprised of TS patients who presented $\mathrm{DCI}>80 \%$. NCs were defined as individuals who had neither chronic diseases nor psychiatric disorders; they were matched for age, sex and ethnic group.

\section{Sample processing}

Peripheral blood samples $(3 \mathrm{ml})$ from patients and controls were drawn in the morning, using a butterfly device inserted into collection tubes equipped with Clot activator and gel for serum separation (BD Biosciences). Collection tubes were treated according to current procedures for clinical samples [60]. To separate serum from cellular components, tubes were rotated end-overend at $20{ }^{\circ} \mathrm{C}$ for 30 ; they were then centrifuged at $4000 \mathrm{rpm}$ in a Beckman J2-21, at $4{ }^{\circ} \mathrm{C}$ for 15 , to spin down blood cells. Supernatant was isolated and centrifuged again at $5000 \mathrm{rpm}$, at $4{ }^{\circ} \mathrm{C}$ for $10^{\prime}$, to remove circulating cells or debris [61]. Serum samples were aliquoted into $1.5 \mathrm{~mL}$, RNase-free, Eppendorf tubes and stored at $-80{ }^{\circ} \mathrm{C}$ until analysis. Sera were analyzed with a Multiscan Ascent microplate reader spectrometer (Thermo Fisher Scientific) at $\lambda=n m 414$, setting an absorbance value $<0.2$ as cut-off [61], to distinguish haemolyzed from non-haemolyzed sera.

\section{RNA extraction}

RNA was extracted from $400 \mu \mathrm{l}$ serum samples by using a Qiagen miRNeasy Mini Kit (Qiagen, GmbH, Hilden, Germany), according to Qiagen Supplementary Protocol for purification of small RNAs from serum and plasma. RNA was eluted in a $40 \mu \mathrm{l}$ total volume of elution buffer 
with two consecutive steps of elution ( $30 \mathrm{ul}$ followed by other $10 \mu \mathrm{l}$ ) in the same collection tube. RNAs were quantified by spectrophotometry.

\section{miRNAs profiling}

To profile the transcriptome of 754 different human miRNAs, $4.5 \mu \mathrm{l}$ of RNA (corresponding to $30 \mathrm{ng}$ of RNA) were retrotranscribed and preamplified according to manufacturer's instructions. Preamplified products were loaded on TLDA, TaqMan Human MicroRNA Array v3.0 A and B (Applied Biosystems, Foster City, CA). PCR reactions on TLDA were performed on a 7900HT Fast Real Time PCR System (Applied Biosystems). To obtain an accurate miRNA profiling, we used the global median normalization (GMN) method. Similar to microarray analysis, $\mathrm{Ct}$ values from each sample were normalized to the median $\mathrm{Ct}$ of the array $[62,63]$. By computing the Pearson correlation among the $\mathrm{Ct}$ medians and means of each array and $\mathrm{Ct}$ of each miRNA, we identified a miRNA that showed an expression profile closer to the median and mean of TLDAs: miR-320. Expression fold changes were calculated by the $2-\Delta \Delta C T$ method $[62,63]$. Differentially Expressed (DE) miRNAs were identified by Significance of Microarrays Analysis (SAM) (http://www.tm4.org), applying an unpaired test among $\Delta \mathrm{Ct}$ and using a p-value based on 100 permutations; imputation engine: K-nearest neighbours (10 neighbours); false discovery rate (FDR) $<0.15$.

\section{Validation with single Taqman assays}

Purified RNAs from sera of 52 TS patients and 15 NCs (validation set) were used for miRNA-specific reverse transcription to obtain miRNA-specific cDNAs. RT-PCR analysis was performed by using TaqMan MicroRNA Assays (Applied Biosystems) specific for DE miRNAs and for controls.

\section{Statistical analyses}

All statistical analyses were performed using the SPSS software (Version 22.0, SPSS, Chicago, IL). Parametric independent Student $t$-test and non parametric Wilcoxon test were used to compare miRNAs serum levels between the TS patients and NCs. DCts for miR-429 respect to endogenous control miR-320 were used to generate a Receiver Operating Characteristic (ROC) curve. Area Under the Curve (AUC) and $95 \%$ confidence intervals (95\% CIs) were calculated to assess the accuracy of each parameter (sensitivity and specificity) and to find an appropriate cut-off point. Statistical significance was established at a $p$-value $\leq 0.05$.

\section{Computational Analysis}

Validated targets of miR- 429 were retrieved by miRTarBase (http://mirtarbase.mbc.nctu.edu.tw/). The corresponding biological network was built by retrieving interactome data through MiMi Plugin of cytoscape (http://mimiplugin.ncibi.org/). The tool FatiGO (http:// babelomics3.bioinfo.cipf.es) was applied to determined the statistical overrepresentation for Gene Ontology (GO), KEGG and BioCarta terms of miRNAs targets network.

\section{Additional file}

Additional file 1: Delta $\mathrm{Ct}$ obtained by global median normalization method for TLDA Panel A and B. (XLSX $72 \mathrm{~kb}$ )

\section{Abbreviations}

ADHD: Attention deficit hyperactivity disorder; ASD: Autism spectrum disorders; AUC: Area under the curve; CADS: Conner's ADHD/DSMV-IV scale; CBCL: Child behavior checklist; CDI: Child depression inventory; cmiRNAs: circulating miRNAs; DCl: Diagnostic confidence index; DE: Differentially expressed; FDR: False discovery rate; MASC: Multidimensional anxiety scale for children; NCs: Unaffected controls; OCD: Obsessive compulsive disorder; SD: Standard deviation; TLDA: TaqMan low density array; TS: Tourette syndrome; WISC-III: Wechsler intelligence scale for children III; Y-BOCS: Children's yale- brown obsessive compulsive scale; YGTSS: Yale global tic severity rating scale; YQOL-R: Youth quality of life instrument-research version.

\section{Competing interests}

The authors declare that they have no competing interests.

\section{Authors' contributions}

$\mathrm{MP}$ conceived and coordinated the project with the critical collaboration of RR. MP, RR, MR,CDP, MS designed experiments; CB, MAS, CP, MGG, DB performed them. MAG and PVC carried out patient's recruitment and clinical data analysis. $C B$ and $A C$ performed computational analysis. $M B$ and $A A$ realized statistical analysis. MP, RR, MR, AA wrote the paper. All authors contributed to the critical revision of the data, read and approved the final manuscript.

\section{Acknowledgements}

We dedicate this paper to our senior Colleague and Friend Prof Lorenzo Pavone, one of pioneers of Pediatric NeuroPsychiatry in Italy. MP, RR and AA acknowledge financial support by Ministero dell'Università e della Ricerca Scientifica e Tecnologica (MIUR) and by Università degli Studi di Catania.

\section{Author details}

${ }^{1}$ Section of Child Neurology and Psychiatry, Department of Experimental and Clinical Medicine, University of Catania, Catania, EU, Italy. ${ }^{2}$ BioMolecular, Genome and Complex Systems BioMedicine Unit (BMGS), Section of Biology and Genetics G Sichel, Department of BioMedicine and BioTechnology, University of Catania, Catania, EU, Italy. ${ }^{3}$ Department of Medical and Surgical Sciences and Advanced Technologies GF Ingrassia, University of Catania, Catania, EU, Italy. ${ }^{4}$ Via S Sofia 87, Building C, $2^{\circ}$ floor, room 10, 95123 Catania, EU, Italy.

Received: 22 May 2015 Accepted: 14 July 2015

Published online: 25 July 2015

\section{References}

1. American Psychiatric Association. Diagnostic and Statistical Manual of Mental Disorders. 5th ed. Washington, DC: American Psychiatric Press; 2013.

2. Robertson MM. The prevalence and epidemiology of Gilles de la Tourette syndrome Part 1: The epidemiological and prevalence studies. J Psychosom Res. 2008;65:461-72.

3. Ghanizadeh A, Mosallaei S. Psychiatric disorders and behavioral problems in children and adolescents with Tourette syndrome. Brain Dev. 2009;31:15-9.

4. Khalifa N, von Knorring AL. Tourette syndrome and other tic disorders in a total population of children: clinical assessment and background. Acta Paediatr. 2005;94:1608-14.

5. Müller-Vahl K, Dodel I, Müller N, et al. Health-related quality of life in patients with Gilles de la Tourette's syndrome. Mov Disord. 2010;25:309-14. 
6. Ludolph AG, Roessner V, Münchau A, Müller-Vahl K. Tourette syndrome and other tic disorders in childhood, adolescence and adulthood. Dtsch Arztebl Int. 2012;109:821-29.

7. Rizzo R, Eddy CM, Calí P, Gulisano M, Cavanna AE. Metabolic effects of aripiprazole and pimozide in children with Tourette syndrome. Pediatr Neurol. 2012;47:419-22.

8. Rizzo R, Curatolo P, Gulisano M, Virzì M, Arpino C, Robertson MM. Disentangling the effects of Tourette syndrome and attention deficit hyperactivity disorder on cognitive and behavioral phenotypes. Brain Dev. 2007;29:413-20.

9. Eddy CM, Rizzo R, Gulisano M, Agodi A, Barchitta M, Calì P, et al. Quality of life in young people with Tourette syndrome: a controlled study. J Neurol. 2011;258:291-301.

10. Worbe Y, Gerardin E, Hartmann A, Valabrégue R, Chupin M, Tremblay L, et al. Distinct structural changes underpin clinical phenotypes in patients with Gilles de la Tourette syndrome. Brain. 2010;133:3649-60.

11. Karagiannidis I, Rizzo R, Tarnok Z, Wolanczyk T, Hebebrand J, Nöthen MM, et al. Replication of association between a SLITRK1 haplotype and Tourette Syndrome in a large sample of families. Mol Psychiatry. 2012;17(7):665-8.

12. Axtell MJ, Westholm JO, Lai EC. Vive la différence: biogenesis and evolution of microRNAs in plants and animals. Genome Biol. 2011;12:22.

13. Krol J, Loedige I, Filipowicz W. The widespread regulation of microRNA biogenesis, function and decay. Nat Rev Genet. 2010;11:597-610.

14. Meola N, Gennarino VA, Banfi S. microRNAs and genetic diseases. Pathogenetics. 2009;2:7.

15. Turchinovich A, Cho WC. The origin, function and diagnostic potential of extracellular microRNA in human body fluids. Front Genet. 2014;5:30.

16. Reid G, Kirschner MB, van Zandwijk N. Circulating microRNAs: Association with disease and potential use as biomarkers. Crit Rev Oncol Hematol. 2011;80:193-208.

17. Cortez MA, Bueso-Ramos C, Ferdin J, Lopez-Berestein G, Sood AK, Calin GA MicroRNAs in body fluids - the mix of hormones and biomarkers. Nat Rev Clin Oncol. 2011;8:467-77.

18. Crowley E, Di Nicolantonio F, Loupakis F, Bardelli A. Liquid biopsy: monitoring cancer-genetics in the blood. Nat Rev Clin Oncol. 2013;10:472-84.

19. Mundalil Vasu M, Anitha A, Thanseem I, Suzuki K, Yamada K, Takahashi T, et al. Serum microRNA profiles in children with autism. Mol Autism. 2014;5:40.

20. Rong H, Liu TB, Yang KJ, Yang HC, Wu DH, Liao CP, et al. MicroRNA-134 plasma levels before and after treatment for bipolar mania. J Psychiatr Res. 2011;45:92-5.

21. Shi W, Du J, Qi Y, Liang G, Wang T, Li S, et al. Aberrant expression of serum miRNAs in schizophrenia. J Psychiatr Res. 2012;46:198-204.

22. Li YJ, Xu M, Gao ZH, Wang YQ, Yue Z, Zhang YX, et al. Alterations of serum levels of BDNF-related miRNA sin patients with depression. PLoS One. 2013;8:e63648

23. Kim J, Inoue K, Ishii J, Vanti WB, Voronov SV, Murchison E, et al. A microRNA feedback circuit in midbrain dopamine neurons. Science. 2007:317:1220-4.

24. Cogswell JP, Ward J, Taylor IA, Waters M, Shi Y, Cannon B, et al. Identification of miRNA changes in Alzheimer's disease brain and CSF yields putative biomarkers and insights into disease pathways. J Alzheimers Dis. 2008;14:27-41.

25. Johnson R, Zuccato C, Belyaev ND, Guest DJ, Cattaneo E, Buckley NJ. A microRNA-based gene dysregulation pathway in Huntington's disease. Neurobiol Dis. 2008;29:438-45.

26. Jiao J, Herl LD, Farese RV, Gao FB. MicroRNA-29b Regulates the Expression Level of Human Progranulin, a Secreted Glycoprotein Implicated in Frontotemporal Dementia. PLoS One. 2010;5:e10551.

27. Hansen $T$, Olsen L, Lindow M, Jakobsen KD, Ullum H, Jonsson E. Brain Expressed microRNAs Implicated in Schizophrenia Etiology. PLOS One. 2007;2:e873.

28. Kim AH, Reimers M, Maher B, Williamson V, McMichael O, McClay JL, et al. MicroRNA expression profiling in the prefrontal cortex of individuals affected with schizophrenia and bipolar disorders. Schizophr Res. 2010;124:183-91.

29. Mellios N, Sur M. The emerging role of microRNAs in schizophrenia and autism spectrum disorders. Front Psychiatry. 2012;3:39.

30. Abelson JF, Kwan KY, O'Roak BJ, Baek DY, Stillman AA, Morgan TM, et al. Sequence Variants in SLITRK1 Are Associated with Tourette's Syndrome. Science. 2005:310:317-20.

31. Mitchell PS, Parkin RK, Kroh EM, Fritz BR, Wyman SK, Pogosova-Agadjanyan EL, et al. Circulating microRNAs as stable blood-based markers for cancer detection. Proc Natl Acad Sci U S A. 2008;105:10513-8.
32. Chen $X, B a Y, M a ~ L, C a i X$, Yin $Y$, Wang $K$, et al. Characterization of microRNAs in serum: a novel class of biomarkers for diagnosis of cancer and other diseases. Cell Res. 2008;18:997-1006.

33. Sheinerman KS, Umansky SR. Circulating cell-free microRNA as biomarkers for screening, diagnosis, and monitoring of neurodegenerative diseases and other neurologic pathologies. Front Cell Neurosci. 2013;7:150.

34. Kroh EM, Parkin RK, Mitchell PS, Tewari M. Analysis of circulating microRNA biomarkers in plasma and serum using quantitative reverse transcription-PCR (qRT-PCR). Methods. 2010;50:298-301.

35. Turchinovich A, Weiz L, Langheinz A, Burwinkel B. Characterization of extracellular circulating microRNA. Nucleic Acids Res. 2011;39:7223-33.

36. Hunter MP, Ismail N, Zhang $X$, Aguda BD, Lee EJ, Yu L, et al. Detection of microRNA expression in human peripheral blood microvesicles. PLoS One. 2008;3:e3694.

37. Valadi H, Ekstrom K, Ekström K, Bossios A, Sjöstrand M, Lee JJ, et al. Exosome mediated transfer of mRNAs and microRNAs is a novel mechanism of genetic exchange between cells. Nat Cell Biol. 2007;9:654-9.

38. Korpal M, Lee ES, Hu G, Kang Y. The miR-200 family inhibits epithelialmesenchymal transition and cancer cell migration by direct targeting of E-cadherin transcriptional repressors ZEB1 and ZEB2. J Biol Chem. 2008:283:14910-4.

39. Ragusa M, Majorana A, Banelli B, Barbagallo D, Statello L, Casciano I, et al. MIR152, MIR200B, and MIR338, human positional and functional neuroblastoma candidates, are involved in neuroblast differentiation and apoptosis. J Mol Med (Berl). 2010;88(10):1041-53.

40. Vaishnavi V, Manikandan M, Tiwary BK, Munirajan AK. Insights on the functional impact of microRNAs present in autism-associated copy number variants. PLoS One. 2013;8:e56781.

41. Marrale M, Albanese NN, Cali F, Romano V. Assessing the impact of copy number variants on miRNA genes in autism by Monte Carlo simulation. PLoS One. 2014;9:e90947.

42. Baron-Cohen S, Scahill VL, Izaguirre J, Hornsey H, Robertson MM. The prevalence of Gilles de la Tourette syndrome in children and adolescents with autism: a large scale study. Psychol Med. 1999;29:1151-9.

43. Clarke RA, Lee S, Eapen V. Pathogenetic model for Tourette syndrome delineates overlap with related neurodevelopmental disorders including Autism. Transl Psychiatry. 2012;2:e158.

44. Peng C, Li N, Ng YK, Zhang J, Meier F, Theis FJ, et al. A unilateral negative feedback loop between miR-200 microRNAs and Sox2/E2F3 controls neural progenitor cell-cycle exit and differentiation. J Neurosci. 2012;32:13292-308.

45. Eipper-Mains JE, Kiraly DD, Palakodeti D, Mains RE, Eipper BA, Graveley BR. microRNA-Seq reveals cocaine-regulated expression of striatal microRNAs. RNA. 2011;17:1529-43.

46. Patel C, Cooper-Charles L, McMullan DJ, Walker JM, Davison V, Morton J. Translocation breakpoint at 7q31 associated with tics: further evidence for IMMP2L as a candidate gene for Tourette syndrome. Eur J Hum Genet. 2011;19:634-9.

47. Petek E, Windpassinger C, Vincent JB, Cheung J, Boright AP, Scherer SW, et al. Disruption of a novel gene (IMMP2L) by a breakpoint in 7q31 associated with Tourette syndrome. Am J Hum Genet. 2001;68:848-58.

48. Tossell K, Andreae LC, Cudmore C, Lang E, Muthukrishnan U, Lumsden A, et al. Lrrn1 is required for formation of the midbrain-hindbrain boundary and organiser through regulation of affinity differences between midbrain and hindbrain cells in chick. Dev Biol. 2011:352:341-52.

49. Steeves TD, Fox SH. Neurobiological basis of serotonin-dopamine antagonists in the treatment of Gilles de la Tourette syndrome. Prog Brain Res. 2008;172:495-513.

50. Robertson MM, Eapen V. The National Hospital Interview Schedule for the assessment of Gilles de la Tourette. Int J Methods Psychiatr Res. 1996;6:203-26.

51. Robertson MM, Banerjee S, Kurlan R, Cohen DJ, Leckman JF, McMahon W, et al. The Tourette syndrome diagnostic confidence index: development and clinical associations. Neurology. 1999;10:2108-12.

52. Wechsler D. Wechsler Intelligence Scale for Children. Thirdth ed. San Antonio TX: The Psychological Corporation; 1991.

53. Topolski TD, Edwards TC, Patrick DL. Youth Quality of Life Instruments. US version. User's manual and Interpretation Guide, First version, Seattle Quality of Life Group. University of Washington: Department of Health Services; 2003.

54. March JS, Parker JDA, Sullivan K, Stallings P, Conners CK. The Multidimensional Anxiety Scales for Children (MASC): Factor structure, reliability and validity. J Am Acad Child Adolesc Psychiatry. 1997;36:544-54. 
55. Kovacs M. Children's Depression Inventory (CDI). Italianed. Firenze: Organizzazioni Speciali; 1988.

56. Conners K. Conner's Parents and Teacher Rating Scales. San Antonio, TX: The Psychological Corporation; 1978.

57. Achenbach T. Manual for the Child Behavior Checklist 4-18. Burlington, VT: University Associate on Psychiatry; 1991.

58. Leckman JF, Riddle MA, Hardin MT, Ort SI, Swartz KL, Stevenson J, et al. The Yale Global Tic Severity Scale: Initial testing of a clinician rated scale of tic severity. J Am Acad Child Adolesc Psychiatry. 1989;28:566-77.

59. Scahill L, Riddle MA, McSwiggin-Hardin M, Ort SI, King RA, Goodman WK, et al. Children's Yale-Brown Obsessive Compulsive Scale: reliability and validity. J Am Acad Child Adolesc Psychiatry. 1997;36:844-52.

60. McDonald JS, Milosevic D, Reddi HV, Grebe SK, Algeciras-Schimnich A. Analysis of Circulating MicroRNA Preanalytical and Analytical Challenges. Clin Chem. 2011;57:833-40.

61. Kirschner MB, Kao SC, Edelman JJ, Armstrong NJ, Vallely MP, van Zandwijk $\mathrm{N}$, et al. Haemolysis during Sample Preparation Alters microRNA Content of Plasma. PLoS One. 2011;6:e24145.

62. Ragusa M, Statello L, Maugeri M, Majorana A, Barbagallo D, Salito L, et al. Specific alterations of the microRNA transcriptome and global network structure in colorectal cancer after treatment with MAPK/ERK inhibitors. J Mol Med. 2012;90:1421-38.

63. Ragusa M, Statello L, Maugeri M, Barbagallo C, Passanisi R, Alhamdani MS, et al. Highly skewed distribution of miRNAs and proteins between colorectal cancer cells and their exosomes following Cetuximab treatment: biomolecular, genetic and translational implications. Oncoscience. 2014;1:132-57.

\section{Submit your next manuscript to BioMed Central and take full advantage of:}

- Convenient online submission

- Thorough peer review

- No space constraints or color figure charges

- Immediate publication on acceptance

- Inclusion in PubMed, CAS, Scopus and Google Scholar

- Research which is freely available for redistribution 